Received: 7 December 2017

Accepted: 8 May 2018

Published online: 25 May 2018

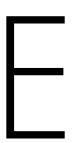

C N
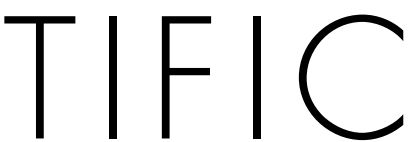

REP

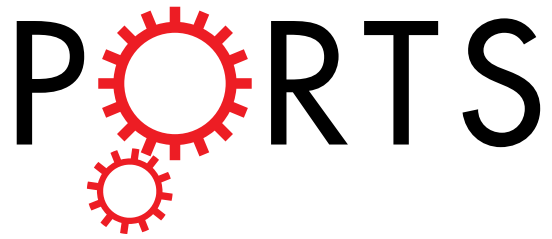

OPEN

\title{
Interfacial engineering of graphene for highly efficient blue and white organic light-emitting devices
}

Shufen Chen ${ }^{1}$, Qin Zhang ${ }^{1}$, Wenjuan Shang ${ }^{1}$, Lihui Liu ${ }^{1}$, Hongtao Yu ${ }^{1}$, Shuai Zhang ${ }^{1}$, Lingling Deng ${ }^{1}$, Min Wang ${ }^{1}$, Minghao Wang ${ }^{1}$, Xue $\mathrm{Li}^{2}$, Baoxiu $\mathrm{Mi}^{1}$ \& Wei Huang ${ }^{1,3}$

Graphene as anodes of flexible organic light-emitting devices (OLEDs) has intrinsic drawbacks of a low work function and a high sheet resistance although it can eliminate the brittle feature of ITO. Chemical doping as a conventional approach is universally used to decrease the sheet resistance and adjust the work function of graphene electrodes, but it suffers from instability problems due to the volatility of chemical species. Here, an insulated poly(4-styrenesulphonate) (PSS) modification layer is firstly coated on the graphene surface along with improved air-stability and hole-injection ability via interfacial dipoles. Besides, the utilization of PSS is beneficial to reduce the leakage current of OLEDs. Then a gradient injection layer of poly(3,4-ethylenedioxythiophene):PSS (PEDOT:PSS)/ tetrafluoroethyleneperfluoro-3,6-dioxa-4-methyl-7-octenesulphonic acid copolymer-doped PEDOT:PSS is covered onto the PSS-modified graphene to further promote hole injection and improve carrier balance inside OLEDs. With above interfacial modification technique, very high efficiencies of $201.9 \mathrm{~cd} \mathrm{~A}^{-1}\left(76.1 \mathrm{~lm} \mathrm{~W} \mathrm{~m}^{-1}, 45.2 \%\right)$ and $326.5 \mathrm{~cd} \mathrm{~A}^{-1}\left(128.2 \mathrm{Im} \mathrm{W}^{-1}, 99.5 \%\right)$ for blue and white emissions are obtained, which are comparable to the most efficient display and lighting technologies so far.

Intrinsic high transparency, remarkable conductivity and wonderful mechanical compliance made graphene an attractive electrode material for applications in flexible optoelectronic and electronic devices, e.g., organic light-emitting devices (OLEDs). The ultimate challenge in developing highly efficient OLEDs with graphene electrodes is universally considered to reduce sheet resistance and tune work function of graphene so as to realize a fine control over the carrier injection and transport ${ }^{1-3}$. Usually, both low sheet resistances and high work functions of graphene were achieved via chemical doping with various acids ${ }^{1,4-6}$, metal halides ${ }^{7-13}$, metal oxides $^{14-16}$ and other organic molecules (e.g., bis(trifluoromethanesulfonyl)amide ${ }^{2}$ or triethyloxonium hexachloroantimonate ${ }^{17}$ ). With these chemical treatments, high luminous efficiencies of both green and white emissions over $250 \mathrm{~cd} \mathrm{~A}^{-1}\left(>160 \mathrm{~lm} \mathrm{~W} \mathrm{~W}^{-1}\right)$ and $120 \mathrm{~cd} \mathrm{~A}^{-1}\left(\sim 90 \mathrm{~lm} \mathrm{~W} \mathrm{~W}^{-1}\right)$ were achieved in graphene-based OLEDs ${ }^{17}$, which are superior to device performances using $\mathrm{ITO}^{18}$, carbon nanotubes ${ }^{19,20}$, metal nanowires ${ }^{21}$, metal grid $^{22}$ and other conductive polymer ${ }^{23}$ anodes. However, some conventional chemical dopants also induce instability problems of graphene and their devices, e.g., gradual decrease of electrical conductivity of graphene due to the volatility of acid $^{24}$ or an increased leakage current due to large metal nanoparticles (Nanoparticles reduced from metal halides usually have sizes of $50-100 \mathrm{~nm})^{25}$. In addition to a direct chemical treatment to graphene, extensive research on hole-injection layer (HIL) has been carried out to improve hole's injection and thus balance carriers in the OLEDs with graphene electrodes. A typical example is doping tetrafluoroethyleneperfluoro3,6-dioxa-4-methyl-7-octenesulphonic acid copolymer (PFI) into the poly(3,4-ethylenedioxythiophene):poly (4-styrenesulphonate) (PEDOT:PSS) with a volume ratio of 1:1, which can significantly enhance holes injection by increasing the graphene's work function to a high level of $5.95 \mathrm{eV}^{1}$. Recently, a sandwiched $\mathrm{TiO}_{2} /$ graphene/ PEDOT:PSS: PFI electrode architecture was used to enhance electroluminescence performance by utilizing cavity resonance effect, yielding an ultrahigh power efficiency of $250 \mathrm{~lm} \mathrm{~W}^{-1}\left(160 \mathrm{~lm} \mathrm{~W}^{-1}\right.$ without a half-ball lens) for

${ }^{1}$ Key Laboratory for Organic Electronics and Information Displays \& Jiangsu Key Laboratory for Biosensors, Institute of Advanced Materials (IAM), Jiangsu National Synergetic Innovation Center for Advanced Materials (SICAM), Nanjing University of Posts \& Telecommunications, 9 Wenyuan Road, Nanjing, 210023, China. ${ }^{2}$ Mechanical Engineering Institute, Nanjing Institute of Technology, Nanjing, 211167, China. ${ }^{3}$ Institute of Flexible Electronics (SIFE), Northwestern Polytechnical University (NPU), 127 West Youyi Road, Xi'an, 710072, Shaanxi, China. Shufen Chen, Qin Zhang, Wenjuan Shang and Lihui Liu contributed equally to this work. Correspondence and requests for materials should be addressed to S.C. (email: iamsfchen@njupt.edu.cn) orW.H. (email: wei-huang@njtech.edu.cn) 

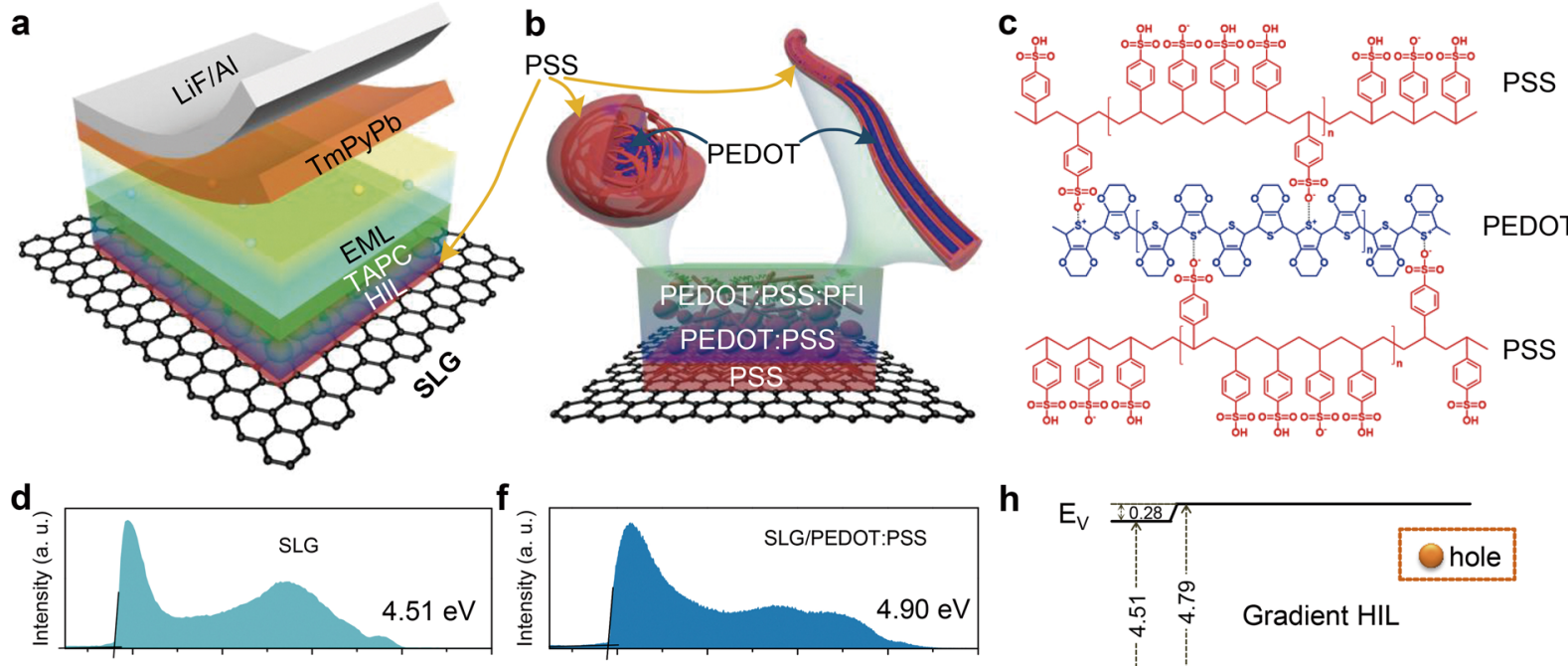

h
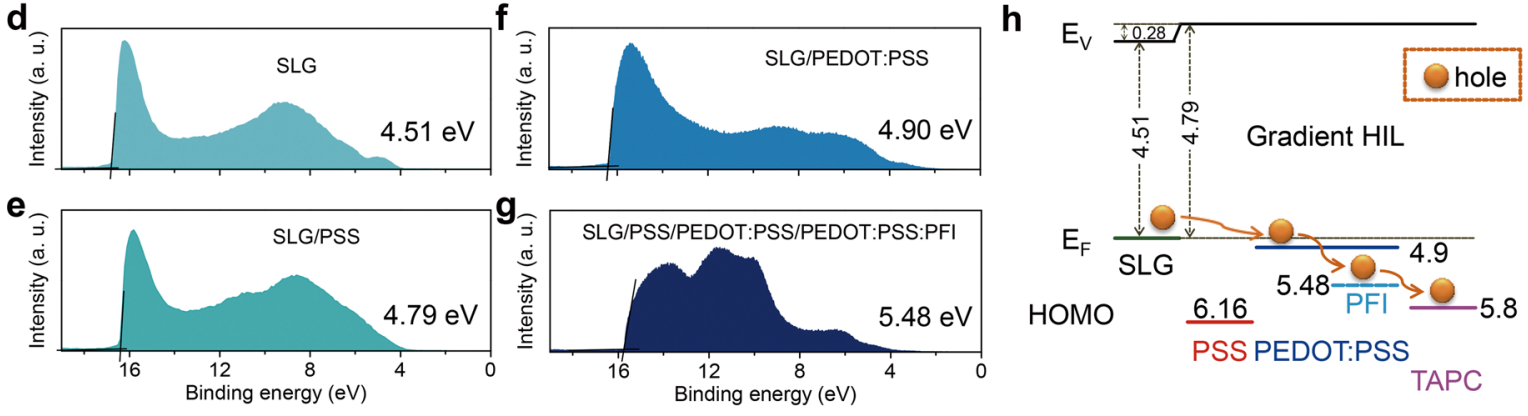

TAPC

Figure 1. (a) Schematic configuration of white OLEDs. (b) Schematic diagram of gradient HIL including PSS, PEDOT:PSS and PFI-doped PEDOT:PSS. Part of the PEDOT:PSS grains (top left) is reformed into nanofibrils (top right) after PFI doping. (c) Chemical bonding of PSS with PEDOT:PSS. (d-g) He I UPS spectra of graphene, PSS, PEDOT:PSS and PFI-doped PEDOT:PSS film samples. The calculated $\mathrm{E}_{\mathrm{F}}$ of graphene and PSS and HOMO levels of PEDOT:PSS and PFI-doped PEDOT:PSS are 4.51, 4.79, 4.90 and 5.48 eV. (h) Energy levels of gradient HIL.

green emission ${ }^{26}$. Unfortunately, this approach is more suitable for design and development of monochromatic OLEDs instead of white ones, due to optical microcavity can only selectively enhance the emission at a certain wavelength by constraining the emissions of other wavelengths ${ }^{27}$.

Herein, we provide with a useful interfacial modification material poly(4-styrenesulphonate) (PSS), which not only reduces hole injection barrier by forming an interfacial dipole on graphene, but also maintains a high optical transmittance $(\sim 97 \%$ at $550 \mathrm{~nm})$ and a good air-stability of the pristine single-layer graphene (SLG). With a further combination of PSS with a PEDOT:PSS/PFI-doped PEDOT:PSS gradient injection layer to sufficiently reduce hole-injection barriers, we notably demonstrate highly efficient white and blue emissions with current (power) efficiencies of $326.5 \mathrm{~cd} \mathrm{~A}^{-1}\left(128.2 \mathrm{~lm} \mathrm{~W}^{-1}\right.$ at $\left.5,270 \mathrm{~cd} \mathrm{~m}^{-2}\right)$ and $201.9 \mathrm{~cd} \mathrm{~A}^{-1}(76.1 \mathrm{~lm} \mathrm{~W}-1$ at $1,494 \mathrm{~cd}$ $\left.\mathrm{m}^{-2}\right)$ in spite of manufacturing devices on moderately conductive SLG anodes $\left(\sim 600 \Omega \mathrm{sq}^{-1}\right)$. This study also indicates that the demanding requirements for the conductivity of graphene can be properly reduced by utilizing a gradient HIL and suitable surface modification, suggesting that a potential large-scale application of graphene in large-size OLED panels or lighting.

\section{Results}

PSS interfacial modification layer. The transfer approach and cleaning process of the SLG sheets used in this work are described in detail in Methods Section. Then the white OLEDs with blue and yellow complementary colors, as the schematic structure illustrated in Fig. 1a-c, are deposited onto SLG sheets via spin-coating or thermal deposition technology. In consideration of the intrinsic hydrophobicity of graphene, the as-transferred SLG is treated with $\mathrm{UV} \mathrm{O}_{3}(6 \mathrm{~min})$ prior to formation of HIL. Then the PSS aqueous solution with an optimal concentration of $3 \times 10^{-6} \mathrm{wt} \%$ is spin-coated onto the SLG at the rotation rate of 3,000 rpm for $60 \mathrm{~s}$, forming an ultrathin modification film of $2.1 \pm 0.2 \mathrm{~nm}$ (Film thickness calculation see Methods Section). Subsequently, PEDOT:PSS and PFI-doped PEDOT:PSS are spin-coated onto the PSS modified graphene surface to comprise the HIL structure proposed in this work.

Interfacial dipole. The influence of PSS on graphene is further confirmed with analysis technique of Raman spectroscopy (Supplementary Fig. S1) and X-ray photoelectron spectroscopy (XPS, Fig. 2). Supplementary Fig. S1f-h reports statistical Raman shifts of over 20 data points for intrinsic and PSS-modified graphene samples. Compared with the intrinsic one, weak Raman shifts of the D, G and 2D band peaks are observed in the modified graphene with a low PSS concentration of $3 \times 10^{-6} \mathrm{wt} \%$. Moreover, the XPS analysis (Fig. 2) indicates that the characteristic $\mathrm{C} 1 \mathrm{~s}$ graphene peak shows no obvious shift towards lower binding energies and it rules out the possibility of doping ${ }^{28}$. The sheet resistance of up to $1,200 \pm 200 \Omega \mathrm{sq}^{-1}$ (Fig. 3g) after PSS modification also demonstrates this point, which is different from the $p$-type doping with universal decrease in sheet resistance ${ }^{1,2,29,30}$. To 

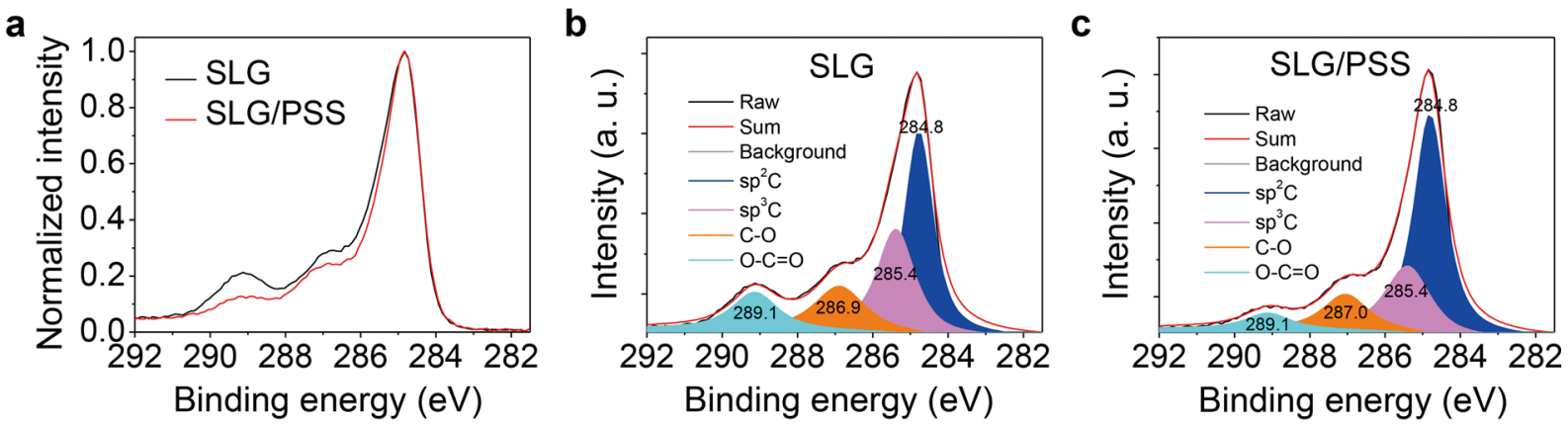

Figure 2. (a) Normalized C1s spectra of graphene sheets with and without PSS modification. The XPS C1s spectra comprised of $s p^{2} \mathrm{C}, s p^{3} \mathrm{C}, \mathrm{C}-\mathrm{O}$ and $\mathrm{O}-\mathrm{C}=\mathrm{O}$ for $(\mathbf{b})$ pristine and (c) PSS-modified graphene.

a

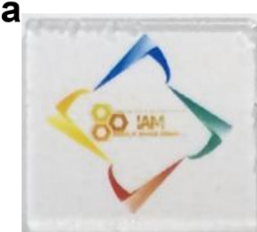

b

F

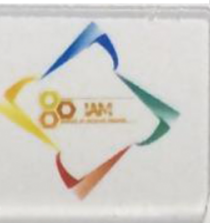

f 100
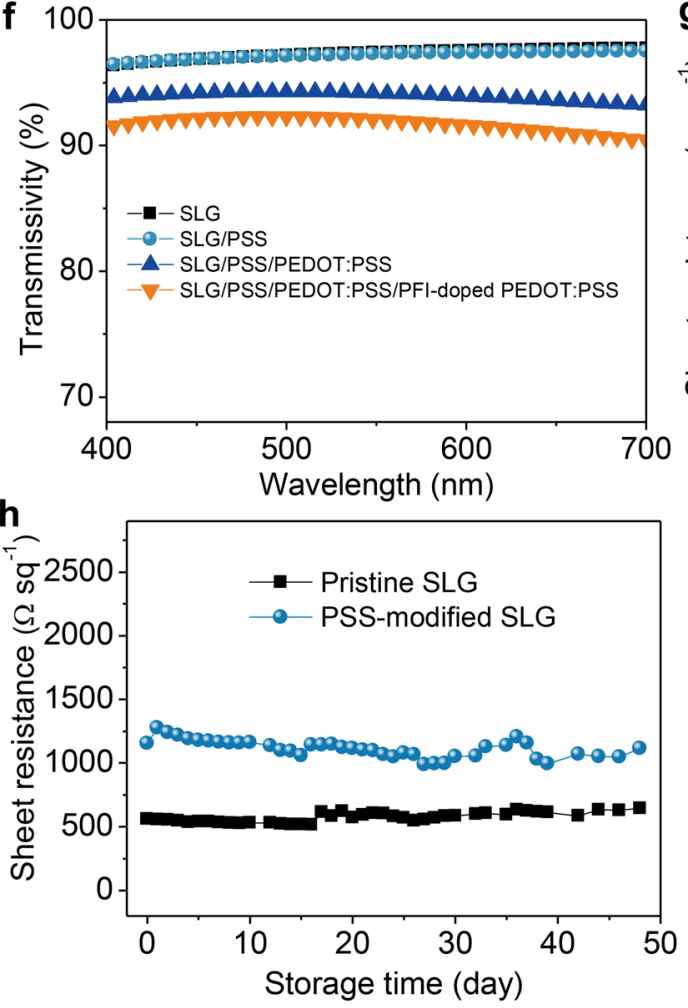

C

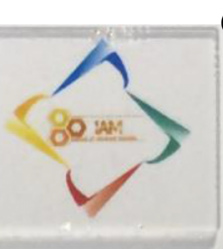

d
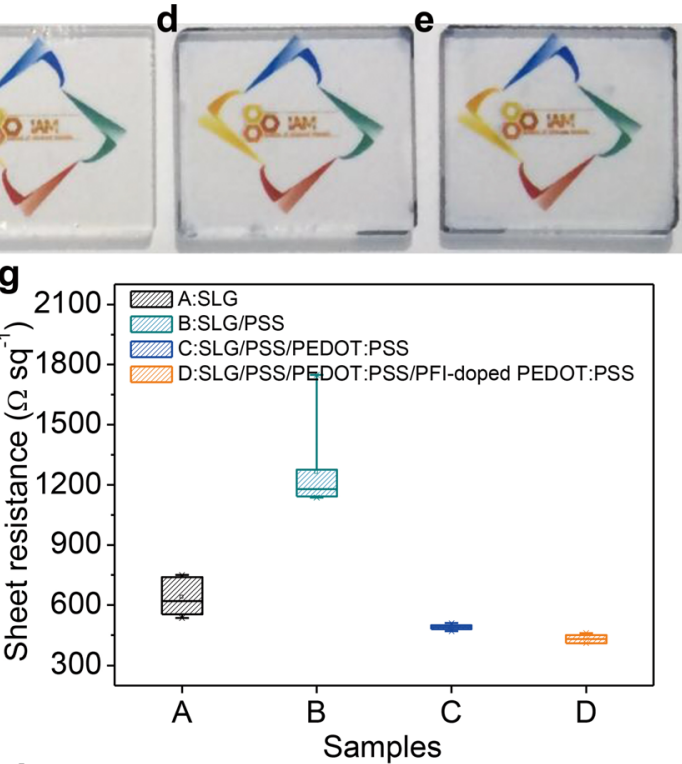

i

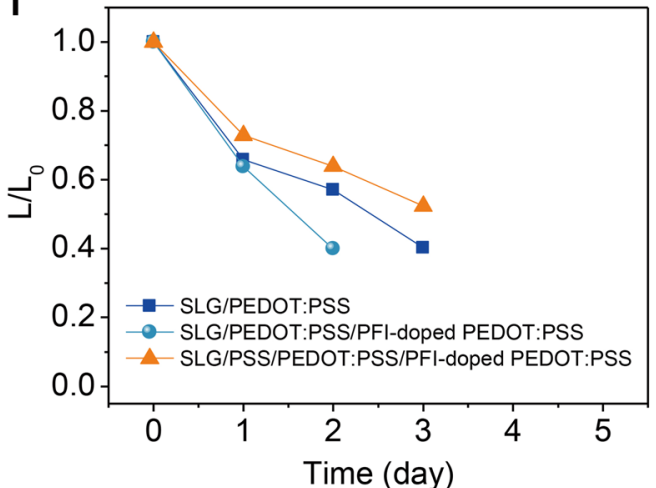

Figure 3. (a-e) Photographs of the glass substrate, monolayer graphene, graphene/PSS, graphene/PSS/ PEDOT:PSS and graphene/PSS/PEDOT:PSS/PFI-doped PEDOT:PSS. (f) Transmittance curves of graphene, graphene/PSS, graphene/PSS/PEDOT:PSS and graphene/PSS/PEDOT:PSS/PFI-doped PEDOT:PSS films. All samples are prepared on quartz substrates. (g) Statistics on sheet resistance of pristine graphene (A), PSSmodified graphene (B), PEDOT:PSS-covered graphene/PSS (C) and PEDOT:PSS:PFI-coated graphene/PSS/ PEDOT:PSS (D). (h) Sheet resistance of pristine graphene and PSS-modified graphene as a function of storage time. These graphene sheets are stored in air. (i) The relative luminance $\left(\mathrm{L} / \mathrm{L}_{0}\right)$ of devices $v s$ time. The devices are aged by storing them without encapsulation in ambient air $\left(25^{\circ} \mathrm{C}\right.$ and a relative humidity of $\left.35 \%\right)$. The initial luminance $\left(\mathrm{L}_{0}\right)$ of these devices is $1000 \mathrm{~cd} \mathrm{~m}^{-2}$. 


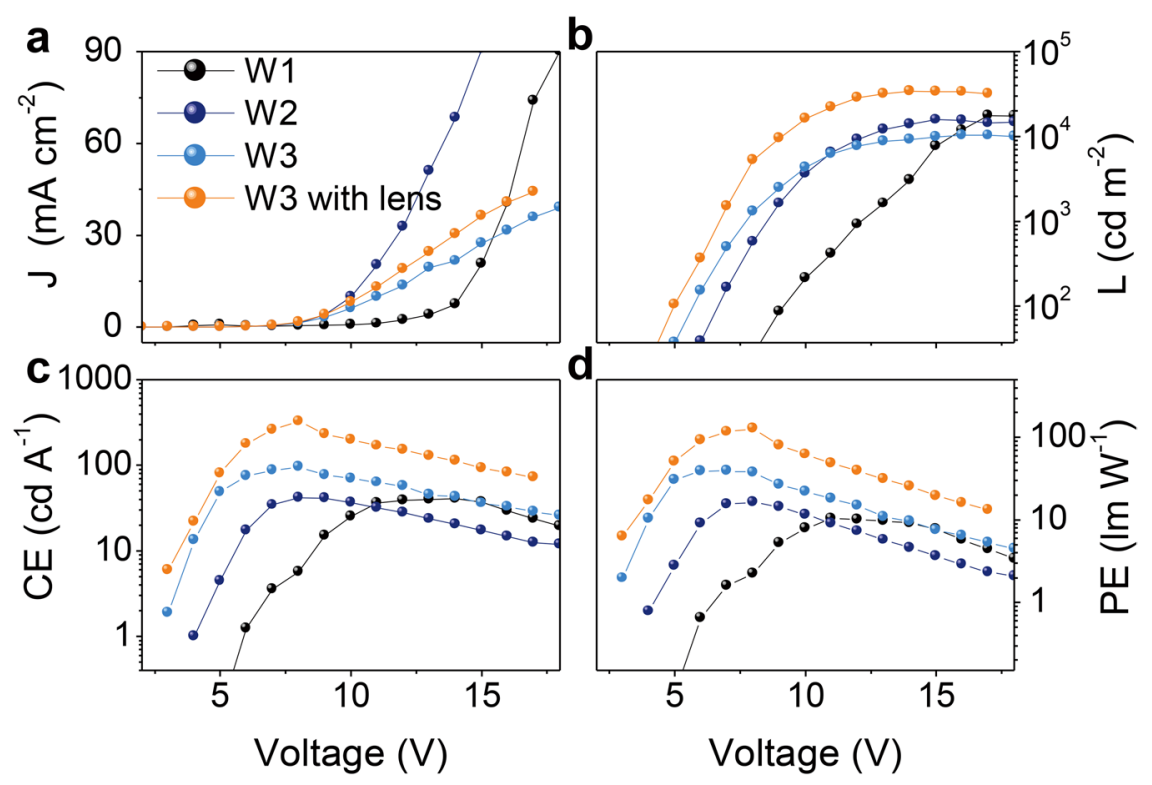

Figure 4. (a-d) The $J-V, \mathrm{~L}, \mathrm{CE}$ and PE curves of $\mathrm{W} 1, \mathrm{~W} 2$ and $\mathrm{W} 3$, respectively. W1, W2 and $\mathrm{W} 3$ correspond to the HIL of pure PEDOT:PSS, the PEDOT:PSS/PFI-doped PEDOT:PSS and the PSS/PEDOT:PSS/PFI-doped PEDOT:PSS. Orange curves describe $J-V, \mathrm{~L}, \mathrm{CE}$ and PE characteristics of W3 with a high refractive index half ball lens on the substrate.

further investigate possible influence of the PSS insulator on graphene's interface energy level, the He I $\alpha$ ultraviolet photoelectron spectroscopy (UPS) spectra of the intrinsic and the PSS-modified graphene are analyzed (Fig. 1d-g). Upon the coating of PSS, the photoemission onset (Fig. 1e) shifts towards a lower binding energy which corresponds to a higher Fermi level $\left(\mathrm{E}_{\mathrm{F}}\right)$ of $4.79 \mathrm{eV}$ ( $4.51 \mathrm{eV}$ for pristine graphene), as the energy level diagram drawn in Fig. $1 \mathrm{~h}$, from which a vacuum level $\left(\mathrm{E}_{\mathrm{V}}\right)$ bending of $\sim 0.3 \mathrm{eV}$ is observed at the graphene/PSS interface induced by interfacial dipoles and this level bending helps to reduce barrier for hole injection. The concept of using insulating materials for controlling the energy level has been commonly used in organic optoelectronic devices with $\mathrm{Al}$ electrodes ${ }^{31-33}$, but few reported in devices with graphene electrodes. Herein, the insulated polymer PSS reduces hole's injection barrier and then improves device's electroluminescent (EL) performances via dipole-induced $\mathrm{E}_{\mathrm{V}}$ bending, instead of forming a p-type doping to graphene.

Film stability. Notably, in contrast with a rapid recovery to high resistance state of pristine graphene for most chemical doping ${ }^{1,24}$, the PSS-modified SLG film in this work shows an excellent air-stability. As illustrated in Fig. $3 \mathrm{~h}$ and Supplementary Fig. S2, by storing these graphene sheets in ambient air, the sheet resistance of the PSS-modified SLG film only exhibits a slight variation in the range of $10 \%$ with the storage time up to 30 days, which performs similar to that of the pristine graphene, indicating an excellent stability of PSS modification on graphene. With increasing measurement times, some local region is destroyed due to multiple contacts of graphene with probes of four-point probe instrument and this leads to a significant increase in sheet resistance of some points, as the statistics shown in Supplementary Fig. S2. In addition, the ultrathin PSS modification layer shows a negligible effect on the transmittance of the SLG film (PSS-coated SLG in Fig. 3c vs pure SLG in Fig. 3b) and a high transmissivity of $97.4 \%$ (at $550 \mathrm{~nm}$ ) is maintained after PSS modification (Fig. 3f), which sufficiently restrains microcavity effect and is particularly beneficial to white light acquirement.

Gradient hole injection layer. High sheet resistance of the PSS-modified SLG $\left(1,200 \pm 200 \Omega \mathrm{sq}^{-1}\right)$ is compensated by the following coatings of PEDOT:PSS and PFI-doped PEDOT:PSS and this value decreases to $490 \pm 20$ and $437 \pm 23 \Omega \mathrm{sq}^{-1}$ (Fig. 3g), respectively, which are comparative with or even slightly lower than those of pristine SLG. Obvious decrease in sheet resistance after the PEDOT:PSS coating is caused by the $p$-type doping of PEDOT:PSS to SLG, while its further decrease with an additional PFI-doped PEDOT:PSS layer is induced by some structural rearrangement of PEDOT:PSS into nanofibrils (Fig. 1b) due to the additive PFI doping ${ }^{34}$. With this PSS interfacial dipole layer and the PEDOT:PSS/PFI-doped PEDOT:PSS bilayer to form a gradient hole injection from 4.51 (pure SLG) to $5.48 \mathrm{eV}$ (See the He I UPS spectra of graphene, PSS, PEDOT:PSS and PFI-doped PEDOT:PSS film samples in Fig. 1d-g), the injection barrier of holes greatly reduces from pure SLG to the hole transport layer 1,1-bis-(4-bis(4-methyl-phenyl)-amino-phenyl)-cyclohexane (TAPC) (with the highest occupied molecular orbital (HOMO) of $5.8 \mathrm{eV}$ ). With this gradient HIL (Fig. 1h), the turn-on voltage $\left(V_{\text {on }}\right)$ in white OLEDs is thus significantly decreased from $5 \mathrm{~V}$ (with PEDOT:PSS) and $4 \mathrm{~V}$ (with PEDOT:PSS/PFI-doped PEDOT:PSS bilayer) to $3 \mathrm{~V}$.

High-performance white OLEDs. As illustrated in Fig. 4a, compared with conventional white OLEDs with PEDOT:PSS as a HIL (W1), the injected current densities are remarkably enhanced in the counterparts with the PEDOT:PSS/PFI-doped PEDOT:PSS bilayer (W2) and the PSS/PEDOT:PSS/PFI-doped PEDOT:PSS 
a

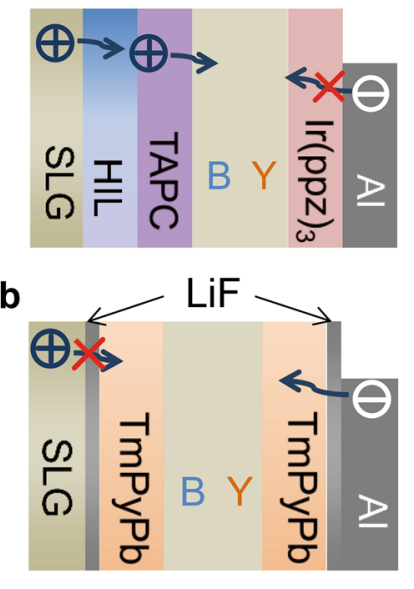

C

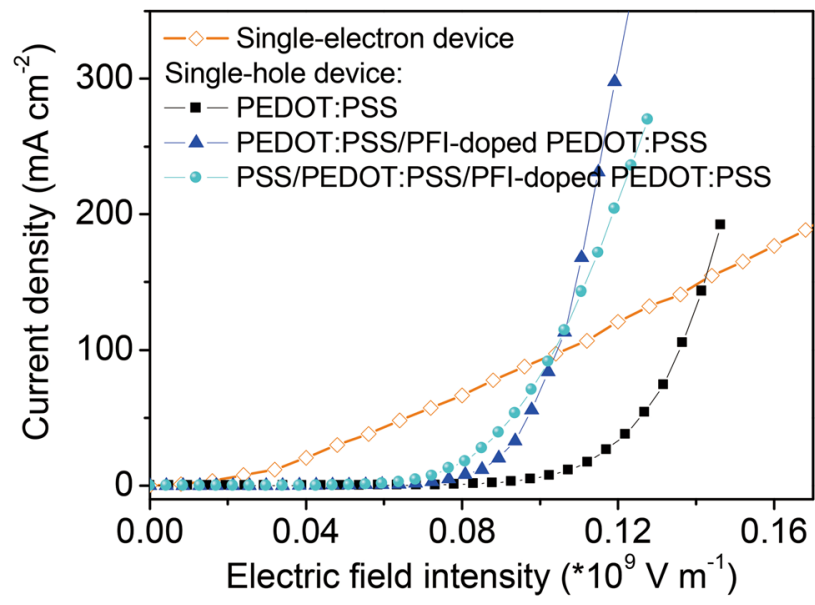

Figure 5. Schematic diagrams of (a) single-hole and (b) single-electron devices. (c) $J$ - $V$ characteristics of single-carrier devices. Note that single-hole devices were respectively fabricated with three different HILs of pure PEDOT:PSS, the PEDOT:PSS/PFI-doped PEDOT:PSS bilayer and the PSS/PEDOT:PSS/PFI-doped PEDOT:PSS trilayer.

trilayer (W3) as the HIL (detailed device structures for white emissions see device fabrication and characterization of Methods Section). Compared with W2, W3 exhibits a decrease in current density and an obvious increase in EL intensity, especially the luminance at low driving voltages. In order to explain this phenomenon, we design single-carrier devices with structures and fabrication process depicted in Methods Section. From the measured current density-voltage $(J-V)$ curves of single-hole and single-electron devices in Fig. 5, we notice that the injected electron current is far larger than the hole counterpart in W1 only using PEDOT:PSS as HIL, and the hole injection is significantly improved with the use of PFI and PSS. In the case of PSS-modified device, the hole current is further improved at an electric field intensity of no more than $10^{8} \mathrm{~V} \mathrm{~m} \mathrm{~m}^{-1}$ (corresponding to a driving bias of less than $12 \mathrm{~V}$ ), indicating a beneficial improvement of the gradient HIL to hole injection. However, we find a larger $J$ in W2 than that in W3, as illustrated in Fig. 4a, which is in contrast with the trend in single-hole devices. And this result suggests a possible large leakage current in W2 due to a weak blocking effect of perfluorinated ionomers PFI on electrons, which does not contribute to performance improvement of white devices, as the luminance (L), current efficiency (CE) and power efficiency (PE) curves shown in Fig. 4b-d and the external quantum efficiency (EQE) curves illustrated in Supplementary Fig. S3a. In contrast, W3 provides a large injection barrier for electrons towards anode due to a wide band gap of the insulating PSS. Thus, W3 shows a maximum CE, PE and EQE of $95.9 \mathrm{~cd} \mathrm{~A}^{-1}(8 \mathrm{~V}), 39.3 \mathrm{~lm} \mathrm{~W}^{-1}(7 \mathrm{~V})$ and $29.2 \%(8 \mathrm{~V})$, exhibiting $2.29,2.40$ and

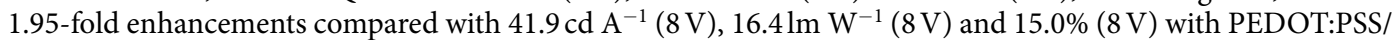
PFI-doped PEDOT:PSS in W2. It is worth mentioning that L in W3 exhibits a 6.45 -fold enhancement at $4 \mathrm{~V}$ and then decreases to 1.18 -fold when the driving voltage increases to $10 \mathrm{~V}$. Note that the maximum L in W3 $(13,420 \mathrm{~cd}$ $\left.\mathrm{m}^{-2} @ 17 \mathrm{~V}\right)$ shows a slight decrease compared with that in $\mathrm{W} 2\left(15,780 \mathrm{~cd} \mathrm{~m}^{-2} @ 15 \mathrm{~V}\right)$ due to a relatively low current. When a high refractive index half ball lens $(n=1.922)$ is covered on the light output side of the W3's glass substrate, very high L, CE, PE and EQE of $34,380 \mathrm{~cd} \mathrm{~m}^{-2}(14 \mathrm{~V}), 326.5 \mathrm{~cd} \mathrm{~A}^{-1}(8 \mathrm{~V}), 128.2 \mathrm{~lm} \mathrm{~W}^{-1}(8 \mathrm{~V})$ and $99.5 \%$ $(8 \mathrm{~V})$ are achieved in $\mathrm{W} 4$, amounting to a $~ 3.7,3.4,3.3$ and 3.4-fold enhancements over luminance, $\mathrm{CE}, \mathrm{PE}$ and EQE in W3. This significant enhancement is due to an improved light outcoupling with a high refractive index hemispherical lens. We must clarify that the optimal EL efficiency in this work is achieved at a high luminance of $5,270 \mathrm{~cd} \mathrm{~m}^{-2}$, different from reported results in literature, which is usually obtained at a low brightness and significantly decreases with driving bias. To the best of our knowledge, up to now, the maximum luminous efficiency of white OLEDs is $\sim 130 \mathrm{~cd} \mathrm{~A}^{-1}$ and $123.4 \mathrm{~lm} \mathrm{~W}^{-1}$ at a luminance of $1,000 \mathrm{~cd} \mathrm{~m}^{-2}$, reported by Tang et al..$^{35,36}$, and it decreases to $106.5 \mathrm{~lm} \mathrm{~W}^{-1}$ when $\mathrm{L}$ is up to $5,000 \mathrm{~cd} \mathrm{~m}^{-2}$. Our results demonstrate a potential of graphene anodes for applications in white OLED lighting. In addition to synthesizing high quality graphene and developing efficient transfer method, graphene modification and device structure design also play an important role in fabricating high efficient OLEDs. The white emission of W3 in this paper exhibits a stable pure white color with a correlated color temperature of 4,200 $\pm 100 \mathrm{~K}$ and a Commission International de L'Eclairage coordinate (CIE) change within $(0.029,0.014)$ at a luminance range of $300-10,000 \mathrm{~cd} \mathrm{~m}^{-2}$ (See the EL spectra in Supplementary Fig. S3d and data collection in Table 1).

High-performance blue OLEDs. Totally different from a microcavity resonance-enhanced approach to enhance EL efficiency ${ }^{26}$, the method in this work is irrelative to the emission color and suitable for fabrication of both monochromatic and white OLEDs. To verify this, blue emission OLEDs are manufactured with our interfacial modification layer and gradient HIL structure, with device configuration described in Methods Section. Similar enhancement effects are observed in these blue emission devices with iridium(III)bis[4,6-(di-fluoropheny 1)-pyridinato-N, $\left.\mathrm{C}^{2}\right]$ picolinate (FIrpic) phosphors. The maximum $\mathrm{CE}, \mathrm{PE}$ and EQE achieve $73.8 \mathrm{~cd} \mathrm{~A}^{-1}$ (at $866 \mathrm{~cd}$ $\left.\mathrm{m}^{-2}\right), 26.91 \mathrm{~m} \mathrm{~W}^{-1}\left(\right.$ at $\left.97 \mathrm{~cd} \mathrm{~m}^{-2}\right), 16.5 \%\left(\right.$ at $\left.866 \mathrm{~cd} \mathrm{~m}^{-2}\right)$ and $201.9 \mathrm{~cd} \mathrm{~A}^{-1}\left(\right.$ at $\left.1,494 \mathrm{~cd} \mathrm{~m}^{-2}\right), 76.1 \mathrm{~lm} \mathrm{~W}^{-1}($ at $896 \mathrm{~cd}$ $\left.\mathrm{m}^{-2}\right), 45.2 \%\left(\right.$ at $1,494 \mathrm{~cd} \mathrm{~m}^{-2}$ ) for blue emissions without and with a high refractive index hemispherical lens, 


\begin{tabular}{|c|c|c|c|c|c|c|c|}
\hline \multicolumn{2}{|l|}{ Device } & \multirow{2}{*}{$\begin{array}{l}V_{\text {on }}[\mathrm{V}]^{\mathrm{a}} \\
5.0\end{array}$} & \multirow{2}{*}{$\begin{array}{l}\begin{array}{l}\mathbf{L}_{\max } @ V \\
\left.\text { [cd m }^{-2} @ \mathbf{V}\right]^{\mathbf{b}}\end{array} \\
17,630 @ 17 \\
\end{array}$} & \multirow{2}{*}{\begin{tabular}{|l|}
$\begin{array}{l}\mathbf{C E}_{\max } ; \mathbf{P E}_{\max } \\
{\left[\mathbf{c d} \mathbf{A}^{-1} ; \mathbf{l m ~ W}^{-1}\right]^{\mathbf{c}}}\end{array}$ \\
$40.8 ; 10.4$ \\
\end{tabular}} & \multirow{2}{*}{$\begin{array}{l}\text { CIE }(x, y) @ 300 \\
\left.\text { [cd m }^{-2}\right]^{d}\end{array}$} & \multirow{2}{*}{\begin{tabular}{|l|} 
CIE $(\mathbf{x}, \mathbf{y})$ \\
$@ 55,000\left[\mathbf{c d ~ m}^{-2}\right]$ \\
$(0.350,0.450)$
\end{tabular}} & \multirow{2}{*}{ 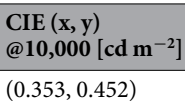 } \\
\hline \multirow{4}{*}{ White } & W1 & & & & & & \\
\hline & W2 & 4.0 & 15,780@15 & $41.9 ; 16.4$ & $(0.339,0.440)$ & $(0.361,0.450)$ & $(0.363,0.452)$ \\
\hline & W3 & 3.0 & 13,420@17 & $95.9 ; 39.3$ & $(0.337,0.440)$ & $(0.365,0.453)$ & $(0.366,0.454)$ \\
\hline & W3 with lens & 2.8 & 34,380@14 & $326.5 ; 128.2$ & $(0.340,0.441)$ & $(0.366,0.453)$ & $(0.365,0.454)$ \\
\hline \multirow{2}{*}{ Blue } & B3 & 3.7 & 6,477@15 & $73.8 ; 26.9$ & $(0.161,0.354)$ & $(0.167,0.367)$ & \\
\hline & B3 with lens & 3.6 & 13,283@15 & $201.9 ; 76.1$ & $(0.161,0.353)$ & $(0.162,0.355)$ & \\
\hline
\end{tabular}

Table 1. Summarized device performances. ${ }^{a} V_{\text {on }}$ : turn-on voltage; ${ }^{b} L_{\text {max }}$ : the maximum luminance; ${ }^{c} \mathrm{CE}_{\max }$, $\mathrm{PE}_{\max }$ : the maximum current and power efficiency; ${ }^{\mathrm{d}} \mathrm{CIE}(\mathrm{x}, \mathrm{y})$ : Commission International de L'Eclairage coordinate.

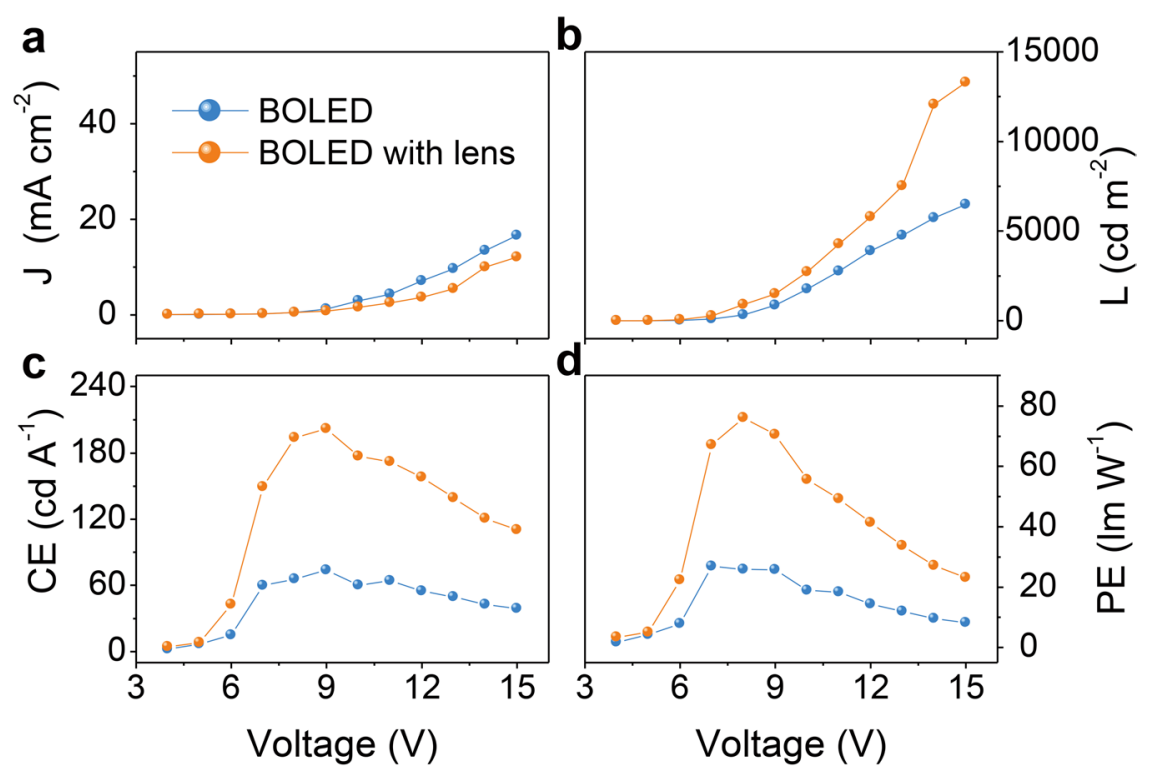

Figure 6. (a) $J-V$, (b) L, (c) $\mathrm{CE}$ and (d) PE curves of $\mathrm{B} 3$ with and without half ball lens.

respectively (Fig. 6, Supplementary Fig. S4a and Table 1). The blue emission also shows a negligible alteration of spectra at a large bias range of $4-15 \mathrm{~V}$ regardless of high refractive index hemispherical lens (Supplementary Fig. S4b and Table 1).

Device stability. Lifetime of blue OLEDs is measured to observe device stability after PSS modification. Note that the devices in this work are aged by storing them without encapsulation in ambient air $\left(25^{\circ} \mathrm{C}\right.$ and a relative humidity of 35\%) and are measured once each day. Tested for 72 hours, the devices with the PSS/PEDOT:PSS/PFI-doped PEDOT:PSS gradient HIL remain $\sim 52 \%$ of an initial luminance $\left(1000 \mathrm{~cd} \mathrm{~m}^{-2}\right)$, while the luminance decreases to $\sim 40 \%$ in the control one with the PEDOT:PSS HIL only (Fig. 3i). Above results demonstrate that the PSS-modified devices show a slight better stability than the control one without any modification, which is mainly due to a good air-stability of the PSS-modified SLG film (Fig. 3h) and a reduction of leakage current inside the OLEDs with the use of PSS ${ }^{37}$.

\section{Discussion}

Graphene as a novel electrode material with excellent transparency, conductivity and mechanical compliance, still faces enormous obstacles for successful applications in flexible OLEDs. These challenges include graphene's surface modification with long-term stability, large area transfer technology of graphene, and encapsulation of OLEDs based on graphene electrodes. This work presents a stable interface modification method to graphene with an insulated PSS, with the formation of a vacuum energy level bending due to the interfacial dipole at the interface of graphene and HIL. By combining this interface modification technique with a gradient hole injection layer, highly efficient blue and white OLEDs with current (power and external quantum) efficiencies of $201.9 \mathrm{~cd}$ $\mathrm{A}^{-1}\left(76.1 \mathrm{~lm} \mathrm{~W}^{-1}\right.$ and $\left.45.2 \%\right)$ and $326.5 \mathrm{~cd} \mathrm{~A}^{-1}\left(128.2 \mathrm{~lm} \mathrm{~W}^{-1}\right.$ and $\left.99.5 \%\right)$ are achieved, respectively. In addition to high efficiency, the white emission also exhibits excellent color temperature and color stability, and all these parameters are sufficient for general display and lighting applications. The understanding gained from this study is expected to allow for the construction of high-performance optoelectronic devices that could find new applications in the fields of perovskite light-emitting diodes, solar cells, thin-film transistors, and potentially many others employing graphene-based electrodes. 


\begin{abstract}
Methods
Graphene transfer. The as-used SLG was synthesized with a chemical vapor deposition process using copper foils as substrates. Transfer method of SLG was referred to our previous work ${ }^{38}$. It is worth noting that the SLG was washed with hot acetone $\left(60^{\circ} \mathrm{C}\right)$ to sufficiently remove surface poly(methyl methacrylate) residue and this step was repeated at least three times, which can eliminate some abnormal electrical phenomena ${ }^{39}$.
\end{abstract}

Film characterization. The transmittance and sheet resistance of as-transferred SLG were measured with an ultraviolet-visible spectrophotometer (Shimadzu, UV-3600) and a four-point probe (RTS-9, China). The components of graphene were analyzed with Raman spectrometer (Reinshaw, InVia). The work function of graphene and the $\mathrm{E}_{\mathrm{F}}$ of each HIL were measured with UPS (Kratos Axis Ultra ${ }^{\alpha}$ ultrahigh vacuum surface analysis system) and these values were calculated by using the photoemission onset to subtract the excitation energy of $21.22 \mathrm{eV}$. The C1s spectra of graphene sheets with and without PSS modification were measured with XPS (Kratos Axis Supra). Film thicknesses except an ultrathin PSS were directly measured with a Stylus Profiler (Bruker, DektakXT). The thickness for the ultrathin PSS interfacial modification layer was inferred with an equation of $I / I_{0}=e^{-\alpha x}$ following the steps below: First, the thicknesses $(x)$ and transmissivities $\left(I / I_{0}\right)$ of several groups of different PSS film thicknesses were measured with a Stylus Profiler and an ultraviolet-visible spectrophotometer to extract the PSS film's absorption coefficient $(\alpha)$. Then, the transmissivity of the PSS ultrathin film used in this work (spin-coating the PSS aqueous solution with $3 \times 10^{-6} \mathrm{wt} \%$ concentration with a rotation ratio of $3,000 \mathrm{rpm}$ for $60 \mathrm{~s}$ to form the ultrathin film) was measured. The thickness of the PSS ultrathin film was eventually calculated with above $\alpha$ and transmissivity, with an average value of $2.1 \pm 0.2 \mathrm{~nm}$.

Device fabrication and characterization. The as-transferred SLG sheets were dried on a hot plate at $120^{\circ} \mathrm{C}$ for $30 \mathrm{~min}$ before device fabrication. Afterwards, the graphene-covered substrates were treated with $\mathrm{UV} \mathrm{O}_{3}$ for $6 \mathrm{~min}$ and then transferred onto a spin-coater to spin-coat the PEDOT:PSS (W1), the PEDOT:PSS/ PFI-doped PEDOT:PSS bilayer (W2) and the PSS/PEDOT:PSS/PFI-doped PEDOT:PSS trilayer (W3) as a HIL. Here, PEDOT:PSS was brought from Heraeus (Clevios ${ }^{\text {TM }}$ P VP AI 4083) with a resistivity of 500-5,000 $\Omega$ $\mathrm{cm}$. After a dry process at $120^{\circ} \mathrm{C}$ for $30 \mathrm{~min}$, the substrates were loaded into a vacuum chamber to thermally deposit the hole transport layer TAPC, the emitting layer (EML), the electron transport layer 1,3,5-tri(m-pyrid3-yl-phenyl)benzene (TmPyPb, $50 \mathrm{~nm})$, the electron injection layer $\mathrm{LiF}(0.5 \mathrm{~nm})$ and the cathode $\mathrm{Al}(100 \mathrm{~nm})$ in sequence, forming final devices with an emitting area of $0.05 \mathrm{~cm}^{-2}$. The EML in white OLEDs is composed of a 19-nm blue light layer and a 1-nm orange-yellow ultrathin one via doping $8 \mathrm{wt} \%$ FIrpic and $10 \mathrm{wt} \%$ Iridium(III)bis(4-phenylthieno[3,2-c] pyridinato- $N, \mathrm{C}^{2}$ ) acetylacetonate (PO-01) phosphorescent guests into the $N, N^{\prime}$-dicarbazolyl-3,5-benzene (mCP) host. While for the blue OLEDs, the EML is comprised of $30 \mathrm{~nm}$ FIrpic (8 wt\%)-doped mCP. Blue emission with the trilayer HIL of PSS/PEDOT:PSS/PFI-doped PEDOT:PSS was denoted as B3, and the parameters of B3 with and without lens were summarized in Table 1. All inorganic/organic layers were thermally deposited in a high-vacuum system with a pressure of less than $5 \times 10^{-4} \mathrm{~Pa}$ and deposition rates of $0.5-10 \AA \mathrm{s}^{-1}$ (5-10 $\mathrm{s}^{-1}$ for $\mathrm{Al}$ and $0.5-1 \AA \mathrm{s}^{-1}$ for other inorganic/organic layers).

Single-hole and single-electron devices with the device configurations of SLG/HIL/TAPC $(40 \mathrm{~nm}) / \mathrm{mCP}$ : $8 \mathrm{wt} \%$ FIrpic $(19 \mathrm{~nm}) / \mathrm{mCP}: 10 \mathrm{wt} \%$ PO-01 $(1 \mathrm{~nm}) /$ tris (phenypyrazole)iridium $\left(\operatorname{Ir}(\mathrm{ppz})_{3}, 100 \mathrm{~nm}\right) / \mathrm{Al}(100 \mathrm{~nm})$ and SLG/LiF $(0.5 \mathrm{~nm}) / \mathrm{TmPyPb}(50 \mathrm{~nm}) / \mathrm{mCP}: 8 \mathrm{wt} \%$ FIrpic $(19 \mathrm{~nm}) / \mathrm{mCP}: 10 \mathrm{wt} \% \mathrm{PO}-01(1 \mathrm{~nm}) / \mathrm{TmPyPb}$ $(50 \mathrm{~nm}) / \mathrm{LiF}(0.5 \mathrm{~nm}) / \mathrm{Al}(100 \mathrm{~nm})$ were fabricated via spin-coating or thermal deposition process similar to the aforementioned OLEDs. Here, the HILs are PEDOT:PSS (1,500 rpm for $60 \mathrm{~s}, 45 \mathrm{~nm})$, PEDOT:PSS (1,500 rpm for $60 \mathrm{~s}, 45 \mathrm{~nm}) / \mathrm{PFI}$-doped PEDOT:PSS (3,000 rpm for $60 \mathrm{~s}, 30 \mathrm{~nm})$ and PSS $\left(3 \times 10^{-6} \mathrm{wt} \%, 3,000 \mathrm{rpm}\right.$ for $\left.60 \mathrm{~s}\right) /$ PEDOT:PSS (1,500 rpm for $60 \mathrm{~s}, 45 \mathrm{~nm}$ )/PFI-doped PEDOT:PSS (3,000 rpm for $60 \mathrm{~s}, 30 \mathrm{~nm})$, which corresponds to devices W1, W2 and W3, respectively. Current density $v s$ electric field intensity characteristics were drawn to ensure that current densities were compared under the same electric field intensity (Fig. 5). Note that the doping ratio of PFI to PEDOT:PSS was 1:2 in volume for all devices.

The EL characteristics were measured with a PR655 spectrometer and a Keithley 2400 programmable voltage-current source. The light output was improved by covering $5 \mathrm{~mm}$ diameter half ball lens with a refractive index of 1.922 onto the glass substrates. All devices including blue OLEDs for lifetime measurements were directly operated at room temperature in ambient air without encapsulation.

Data availability. All data generated or analyzed during this study are available from the corresponding author.

\title{
References
}

1. Han, T.-H. et al. Extremely efficient flexible organic light-emitting diodes with modified graphene anode. Nat. Photon. 6, 105-110 (2012).

2. Kim, D., Lee, D., Lee, Y. \& Jeon, D. Y. Work-function engineering of graphene anode by bis(trifluoromethanesulfonyl)amide doping for efficient polymer light-emitting diodes. Adv. Funct. Mater. 23, 5049-5055 (2013).

3. Zhu, X.-Z. et al. The application of single-layer graphene modified with solution-processed $\mathrm{TiO}_{\mathrm{x}}$ and PEDOT:PSS as a transparent conductive anode in organic light-emitting diodes. Org. Electron. 14, 3348-3354 (2013)

4. Han, T. H. et al. Approaching ultimate flexible organic light-emitting diodes using a graphene anode. NPG Asia Mater. 8, e303 (2016).

5. Liu, Z. K. et al. The application of highly doped single-layer graphene as the top electrodes of semitransparent organic solar cells. ACS Nano 6, 810-818 (2012).

6. Han, T. H. et al. Versatile p-type chemical doping to achieve ideal flexible graphene electrodes. Angew. Chem. Int. Ed. 55, 6197-6201 (2016).

7. Bointon, T. H. et al. Large-area functionalized CVD graphene for work function matched transparent electrodes. Sci. Rep. 5, 16464 (2015).

8. Shin, H. J. et al. Control of electronic structure of graphene by various dopants and their effects on a nanogenerator. J. Am. Chem. Soc. 132, 15603-15609 (2010)

9. Khrapach, I. et al. Novel highly conductive and transparent graphene-based conductors. Adv. Mater. 24, 2844-2849 (2012). 
10. Shin, D. H. et al. Graphene/porous silicon Schottky-junction solar cells. J. Alloy. Compd. 715, 291-296 (2017).

11. Kwon, K. C., Choi, K. S. \& Kim, S. Y. Increased work function in few-layer graphene sheets via metal chloride doping. Adv. Funct. Mater. 22, 4724-4731 (2012).

12. Kang, M. H., Milne, W. I. \& Cole, M. T. Temporal stability of metal-chloride-doped chemical-vapour-deposited graphene. ChemPhysChem 17, 2545-2550 (2016).

13. Kwon, K. C., Choi, K. S., Kim, C. \& Kim, S. Y. Effect of transition-metal chlorides on graphene properties. Phys. Status Solidi A 211, 1794-1800 (2014).

14. Chen, Z. Y. et al. Surface transfer hole doping of epitaxial graphene using $\mathrm{MoO}_{3}$ thin film. Appl. Phys. Lett. 96, 213104 (2010).

15. Kuruvila, A. et al. Organic light emitting diodes with environmentally and thermally stable doped graphene electrodes. J. Mater. Chem. C 2, 6940-6945 (2014).

16. Meng, H. et al. Top-emission organic light-emitting diode with a novel copper/graphene composite anode. Adv. Funct. Mater. 23, 3324-3328 (2013).

17. Li, N. et al. Efficient and bright organic light-emitting diodes on single-layer graphene electrodes. Nat. Commun. 4, 2294 (2013).

18. Wang, J. X. et al. Simple-structured phosphorescent warm white organic light-emitting diodes with high power efficiency and low efficiency roll-off. ACS Appl. Mater. Interfaces 8, (10093-10097 (2016).

19. Yu, L. P., Shearer, C. \& Shapter, J. Recent development of carbon nanotube transparent conductive films. Chem. Rev. 116, 13413-13453 (2016).

20. Li, L. et al. A solution processed flexible nanocomposite electrode with efficient light extraction for organic light emitting diodes. Sci. Rep. 4, 4307 (2014).

21. Gaynor, W. et al. Color in the corners: ITO-free white OLEDs with angular color stability. Adv. Mater. 25, 4006-4013 (2013).

22. Xiang, H.-Y. et al. Outcoupling-enhanced flexible organic light-emitting diodes on ameliorated plastic substrate with built-in indium-tin-oxide-free transparent electrode. ACS Nano 9, 7553-7562 (2015).

23. Fehse, K., Walzer, K., Leo, K., Lövenich, W. \& Elschner, A. Highly conductive polymer anodes as replacements for inorganic materials in high-efficiency organic light-emitting diodes. Adv. Mater. 19, 441-444 (2007).

24. Kim, Y. et al. Vapor-phase molecular doping of graphene for high-performance transparent electrodes. ACS Nano 8, 868-874 (2014).

25. Gunes, F. et al. Layer-by-layer doping of few-layer graphene film. ACS Nano 4, 4595-4600 (2010).

26. Lee, J. et al. Synergetic electrode architecture for efficient graphene-based flexible organic light-emitting diodes. Nat. Commun. 7, 11791 (2016).

27. Chen, S. et al. Recent developments in top-emitting organic light-emitting diodes. Adv. Mater. 22, 5227-5239 (2010).

28. Sanders, S. et al. Engineering high charge transfer $\mathrm{n}$-doping of graphene electrodes and its application to organic electronics. Nanoscale 7, 13135-13142 (2015).

29. Medina, H., Lin, Y. C., Obergfell, D. \& Chiu, P. W. Tuning of charge densities in graphene by molecule doping. Adv. Funct. Mater. 21, 2687-2692 (2011).

30. Chen, W., Chen, S., Qi, D. C., Gao, X. Y. \& Wee, A. T. S. Surface transfer p-type doping of epitaxial graphene. J. Am. Chem. Soc. 129, 10418-10422 (2007).

31. Hung, L. S., Tang, C. W. \& Mason, M. G. Enhanced electron injection in organic electroluminescence devices using an $\mathrm{Al} / \mathrm{LiF}$ electrode. Appl. Phys. Lett. 70, 152-154 (1997).

32. Li, F., Tang, H., Anderegg, J. \& Shinar, J. Fabrication and electroluminescence of double-layered organic light-emitting diodes with the $\mathrm{Al}_{2} \mathrm{O}_{3} / \mathrm{Al}$ cathode. Appl. Phys. Lett. 70, 1233-1235 (1997).

33. Mori, T., Fujikawa, H., Tokito, S. \& Taga, Y. Electronic structure of 8 -hydroxyquinoline aluminum/LiF/Al interface for organic electroluminescent device studied by ultraviolet photoelectron spectroscopy. Appl. Phys. Lett. 73, 2763-2765 (1998).

34. Xu, R.-P., Li, Y.-Q. \& Tang, J.-X. Recent advances in flexible organic light-emitting diodes. J. Mater. Chem. C 4, 9116-9142 (2016).

35. Ou, Q.-D. et al. Extremely efficient white organic light-emitting diodes for general lighting. Adv. Funct. Mater. 24, 7249-7256 (2014).

36. Xu, L.-H. et al. Microcavity-free broadband light outcoupling enhancement in flexible organic light-emitting diodes with nanostructured transparent metal-dielectric composite electrodes. ACS Nano 10, 1625-1632 (2016).

37. Kwon, K. C. et al. Extension of stability in organic photovoltaic cells using UV/ozone-treated graphene sheets. Sol. Energy Mater. Sol. Cells 109, 148-154 (2013).

38. Zhou, Y. F. et al. Poly (sodium 4-styrenseulfonate)-modified monolayer graphene for anode applications of organic photovoltaic cells. Appl. Phys. Lett. 111, 113302 (2017).

39. Zhang, Q. et al. Negative differential resistance and hysteresis in graphene-based organic light-emitting devices. J. Mater. Chem. C 6, 1926-1932 (2018).

\section{Acknowledgements}

The authors acknowledge financial support from the National Foundation for Science and Technology Development (973 project, Grant No. 2015CB932203), the National Key Research and Development Program of China (Grant No. 2017YFB0404501), the National Natural Science Foundation of China (Grant Nos 61274065, 61705111, 61474064 and 61505086), the Ministry of Education Humanities and Social Science Research Projects (Grant No. 13YJCZH091), the Science Fund for Distinguished Young Scholars of Jiangsu Province of China (Grant No. BK20160039), the Natural Science Foundation of Jiangsu Province (Grant No. BM2012010), the Priority Academic Program Development of Jiangsu Higher Education Institutions (Grant No. YX030002), the Jiangsu National Synergetic Innovation Center for Advanced Materials, and the Synergetic Innovation Center for Organic Electronics and Information Displays.

\section{Author Contributions}

S.C., Q.Z., W.S. and L.L. contributed equally to this work. S.C. had the idea for the experiments. S.C., B.M. and W.H. conceived the project and wrote the first draft of the manuscript. Q.Z., W.S., L.L. and S.Z. were primarily responsible for the device fabrication and characterizations. Q.Z. and M.W. transferred graphene sheets. Q.Z. conducted the analysis of Raman spectra and lifetime measurements. Q.Z. and H.Y. measured the UPS data. H.Y. drew the images of Fig. 1a,b,c, M.(H.)W. drew the image of Fig. 1h, and S.C. drew the remaining images of the manuscript. L.D. calculated the EQE data. L.L. and X.L. provided valuable advices and revised manuscript. All authors contributed to data analysis, discussions and manuscript preparation.

Additional Information

Supplementary information accompanies this paper at https://doi.org/10.1038/s41598-018-26464-8.

Competing Interests: The authors declare no competing interests. 
Publisher's note: Springer Nature remains neutral with regard to jurisdictional claims in published maps and institutional affiliations.

(c) (i) Open Access This article is licensed under a Creative Commons Attribution 4.0 International License, which permits use, sharing, adaptation, distribution and reproduction in any medium or format, as long as you give appropriate credit to the original author(s) and the source, provide a link to the Creative Commons license, and indicate if changes were made. The images or other third party material in this article are included in the article's Creative Commons license, unless indicated otherwise in a credit line to the material. If material is not included in the article's Creative Commons license and your intended use is not permitted by statutory regulation or exceeds the permitted use, you will need to obtain permission directly from the copyright holder. To view a copy of this license, visit http://creativecommons.org/licenses/by/4.0/.

(C) The Author(s) 2018 